\section{PICU OUTREACH EDUCATION IMPROVES LOCAL MANAGEMENT OF STATUS EPILEPTICUS (SE)}

doi:10.1136/archdischild-2012-302724.0974

C Goedvolk, R Phatak. North West North UK Paediatric Transport Service, Warrington, UK

Background and Aims In september 2011 results of an audit on the acute management of SE in referring hospitals highlighting safety and feasibility of extubation in some children avoiding transfer to PICU were presented. We re-audited practice to determine whether education had an effect on local extubation rates.

Methods Audit of referral forms with a diagnosis of "seizures", "SE", "fit/fitting," "convulsion" and "epilepsy" from 1 September 2011 till 1 April 2012. Review of discharge summaries and notes of patients transferred to PICU.

Results 56 referrals for seizures (48 patients) in the 7 month period. At referral, 49 patients were intubated. 30 of 49 intubated patients were transferred to PICU, 19 extubated locally. 15 of 30 retrieved and 6 of 15 extubated patients had epilepsy.

Reasons for not attempting extubation included: ongoing seizures (2), Petechial rash (2), transfer for expert opinion (1), previous failed extubation (2) and refusal to assess (2).

All patients managed locally extubated within 6 hours. Extubation in PICU was after $<6(4),<12(7),<24$ (11) or $>24$ hours (3), data unavailable in 4.

1 patient was re-intubated locally because of further seizures. Conclusions We found an increase in safe extubations: 18.9\% prior and $38.7 \%$ after. This re-audit indicates that outreach education by PICU retrieval teams can achieve change in practice. It reconfirms that patients with SE transferred to PICU have a short duration of intubation. Refusal to assess feasibility of extubation by the local hospital can no longer be considered good medical practice.

\section{PERIPHERAL TISSUE NIRS OXIMETRY: REPRODUCIBILITY AND DYNAMIC RANGE OF INVOS 5100C, NONIN EQUANOX, AND FORE-SIGHT}

doi:10.1136/archdischild-2012-302724.0975

S Hyttel-Sørensen, T Witzner Hessel, G Greisen. Copenhagen University Hospital, Rigshospitalet, Copenhagen, Denmark

Background and Aims Near infrared spectroscopy (NIRS) could be a valuable tool in the NICU, but implementation into standard clinical care has yet to been seen. Different absolute values and dynamics of different devices and poor reproducibility could be the cause. Present study is a comparison between the adult sensors of INVOS 5100C, FORE-SIGHT and NONIN EQUANOX 7600.

Methods 10 repositionings on the same spot and 10 repostionings on slightly differing spots during steady state on the adult forearm followed by 6 cuff inflations to $250 \mathrm{mmHg}$ and subsequent tissue deoxygenation. Reproducibility was estimated by the within-subject standard deviation, $S_{w}$ and dynamic range by the difference between the pre- and post-cuff inflation $\mathrm{rStO}_{2} \Delta \mathrm{rStO}$

Results 10 adults participated. All with double skinfold less than $10 \mathrm{~mm}$.

Mean $\mathrm{rStO}_{2}$ was 71.1\% (CI 68.4-73.9\%), 68.1\% (CI 65.2$71.0 \%$ ), and $65.1 \%$ (95\% CI 63.3-67.0\%) with INVOS, NONIN, and FORE-SIGHT, respectively. INVOS gave significantly higher values than FORE-SIGHT $(p=0.003)$. All other differences were insignificant.

Reproducibility of FORE-SIGHT was best, while the reproducibility of NONIN was worst (table 1). Same-site reproducibility and various-site reproducibility was equal.

In pairwise comparisons $\Delta \mathrm{rStO}_{2}$ of NONIN, INVOS and FORESIGHT were significantly different (all $\mathrm{p}<0.0001)$ (table 2$)$.
The signal-to-noise ratio, i.e., $\Delta \mathrm{rStO}_{2} / \mathrm{S}_{\mathrm{w}}$ was $17.6,14.5$, and 12.5 for FORE-SIGHT, INVOS and NONIN, respectively.

Conclusion The different absolute values and dynamic ranges will make comparison of data collected with different devices difficult.

\begin{tabular}{|c|c|c|c|c|c|c|}
\hline & \multicolumn{2}{|c|}{ Same site } & \multicolumn{2}{|c|}{ Verious utes } & \multicolumn{2}{|c|}{ Overall } \\
\hline & S.(\%) & $\mathrm{Cl}(\mathrm{s})$ & S.(\$) & $c(s)$ & $S_{0}(8)$ & $\mathrm{Cl}(\boldsymbol{*})$ \\
\hline Evos & 2.9 & 2.4 .33 & 3.6 & 3.0 .4 .1 & 32 & 28.36 \\
\hline SONIN & 46 & 3.9 .53 & 38 & 93.4 & 4.2 & 3.8 .47 \\
\hline FORE-SRCHKT & 20 & $\{.7 .2\}$ & 2.4 & 20.2 .7 & 22 & 20.24 \\
\hline
\end{tabular}

Table 2. Dynamic range of the NIRS devices

\begin{tabular}{|c|c|c|}
\hline & $\Delta \mathrm{rStO}_{2}(\%)$ & $\mathrm{CI}(\%)$ \\
\hline INVOS & 46.6 & $44.9-48.2$ \\
\hline NONIN & 52.7 & $49.9-55.4$ \\
\hline
\end{tabular}

\section{CYCLOSPORINE-ASSOCIATED THROMBOTIC MICROANGIOPATHY AND THROMBOCYTOPENIA- ASSOCIATED MULTIPLE ORGAN FAILURE: A CASE SUCCESSFULLY TREATED WITH THERAPEUTIC PLASMA EXCHANGE}

doi:10.1136/archdischild-2012-302724.0976

${ }^{1} \mathrm{C}$ Ödek, ${ }^{1} \mathrm{~T}$ Kendirli, ${ }^{1} \mathrm{~A}$ Yaman, ${ }^{2} \mathrm{~T}$ I Ileri, ${ }^{3} \mathrm{Z}$ Kuloğlu, ${ }^{3} \mathrm{~A}$ Kansu, ${ }^{1} \mathrm{E}$ Ince. ${ }^{1}$ Pediatric Critical Care; 'Pediatric Hematology; ${ }^{3}$ Pediatric Gastroenterology, Ankara University, Faculty of Medicine, Ankara, Turkey

Introduction Thrombotic microangiopathy (TMA) is characterized by microvascular thrombosis, thrombocytopenia, and microangiopathic hemolytic anemia. Thrombotic thrombocytopenic purpura (TTP), hemolytic-uremic syndrome (HUS), and disseminated intravascular coagulation (DIC) are responsible from most of these cases. Secondary TMA syndromes are associated with sepsis/infection, cancer, transplantation, autoimmune diseases, and drugs. Studies showed that cyclosporine (CSA) is associated with TMA but the number of reported cases are very small.

Case Report A 13-year-old girl was adimitted to the pediatric intensive care unit (PICU) with multiple organ failure. She was diagnosed with polyglandular deficiency syndrome at an outside facility and had a history of celiac disease, autoimmune thyroiditis, and diabetes mellitus type I. CSA was started seven months before our PICU admission for persistent diarrhea. In PICU admission the patient was thrombocytopenic, anemic and she had multiple organ failure (renal, cardiovascular, hepatic, respiratory, and hematologic). Laboratory and clinical findings were consistent with TMA and TAMOF. TTP, HUS, DIC, and sepsis ruled out. We thought that CSA was the cause of TMA and TAMOF. CSA was stopped and five days of therapeutic plasma exchange (TPE) procedure performed. With TPE the patient improved clinically. Laboratory findings were normalized and after five days of TPE, TMA and MOF dissolved.

Conclusion CSA can be associated with TMA and TAMOF. The most commonly used strategy in treatment is the discontinuation of CSA. The experience in this case indicates that TPE may be effective in treatment of CSA-associated TMA and TAMOF especially in the presence of systemic TMA and MOF.

\section{THE CLINICAL CHARACTERISTICS AND MICROSURGERY FOR TUMORS IN THE FOURTH VENTRICLE TUMORS IN CHILDREN}

